\title{
INNOVATION ET SYSTÈMES COMPLEXES
}

Réflexion méthodologique appliquée à la conception de nouvelles formes de savoirs collectifs au service de l'intérêt général, pour penser la complexité avec simplicité

\author{
Auteur : \\ Francis BEAU \\ Docteur en Sciences de l'Information et de la Communication \\ Université Polytechnique Hauts-de-France - Laboratoire DeVisu \\ E-mailfrancisbeau@gmail.com
}

\section{Résumé :}

Partant de cette idée que le progrès doit être méthodologique avant d'être technologique, la démarche scientifique doit pouvoir renouer avec une «épistémè classique » préconisant ordre et méthode, pour relever avec prudence le défi du modernisme. La complexité de l'administration de notre maison commune (l'économie) considérée comme un écosystème de l'innovation peut être abordée comme un problème d'exploitation d'un capital de connaissances à mettre en valeur avec intelligence. Dans le contexte de « révolution culturelle et cognitive » dont l'électronique et le numérique ne sont que des marqueurs technologiques à effet sur l'ensemble du spectre scientifique et, plus largement, sur l'ensemble du fonctionnement complexe de nos sociétés humaines, une «intelligence collective » s'impose, mettant l'information et l'anticipation par l'innovation, au service de la performance collective et du progrès social. Cette « intelligence économique » dont la finalité est de satisfaire l'intérêt général pour l'édification d'un bien commun, peut s'appuyer sur une mémoire collective et un langage documentaire commun qui l'organise, pour produire une pensée systémique collégiale susceptible d'assurer la résilience de sociétés engagées dans une transformation extraordinairement profonde. En regard de la complexité illimitée de son objet, cette pensée est simple : essentiellement pratique, car orientée vers l'action, elle aborde les systèmes dans leur ensemble au travers d'une grille conceptuelle déterminée par la situation et par l'intention collective. Le langage qui la sert dans une mémoire documentaire, pour inscrire l'activité cognitive d'une collectivité dans la durée autour d'un besoin commun qui lui donne sens, peut s'avérer d'un usage aussi courant qu'une langue maternelle, en s'appuyant sur une organisation logique naturelle inspirée des mécanismes sensoriels à l'œuvre dans nos systèmes cognitifs individuels.

\section{Mots-clés :}

méthodologie, épistémè, systèmes cognitifs, transformation digitale, mémoire collective, intelligence sociale, pensée collégiale, intérêt général, anticipation, innovation, progrès, numérique, analogique

Le travail de réflexion qui suit n'a pas pour ambition de contribuer à une «nouvelle théorie de l'innovation », qui aborderait celle-ci sous l'angle de l'artefact technique produit, en analysant sa conception au travers de différents «cadres » d'action, « de fonctionnement » et «d'usage », pour en réaliser «l'alliage» dans un «cadre de référence sociotechnique ( Flichy, 2003). Il ne s'agit pas non plus d'y aborder l'innovation sous le seul angle des progrès techniques dont on étudierait, à la suite de Schumpeter, l'influence sur le comportement des agents économiques, et les répercussions sociales de « destruction créatrice » (Schumpeter, 1943). Il s'agit plutôt, dans le prolongement d'un travail de thèse récent (Beau, 2019), de tenter d'apporter une contribution à la pensée systémique, qui s'insèrerait dans un espace théorique peu exploité, en opérant une sorte d'inversion de la démarche schumpeterienne. C'est en effet un renversement de la perspective qui reconnaitrait cette fois à la méthodologie le pouvoir d'influencer le progrès technique afin de lui donner tout son sens. La méthodologie pourrait ainsi agir 
en amont sur les comportements économiques, en pensant la complexité avec toute la simplicité nécessaire à l'élaboration collective d'un bien commun, pour la satisfaction de l'intérêt général et à terme, la durabilité de notre fragile écosystème.

\section{LA METHODOLOGIE ET L'INNOVATION AU SERVICE DE L'INTERET GENERAL}

Avant toute considération sur l'innovation, il faut accepter l'idée que celle-ci ne se limite pas à la technologie, mais peut et doit surtout venir d'avancées méthodologiques. Ceci est vrai de manière générale, mais plus particulièrement encore, à l'ère de l'économie de la connaissance, en matière d'information. Puissent les décideurs et les responsables des systèmes d'information dans l'entreprise ou dans les administrations, être convaincus du bien-fondé de cette idée saugrenue que l'innovation ne se limite pas aux Nouvelles Technologies (numériques) de l'Information et de la Communication (les NTIC), mais doit avant tout pouvoir s'appuyer sur des développements méthodologiques, qui font appel aux Sciences de l'Information et de la Communication (les SIC) et relèvent ainsi des sciences humaines et sociales. Si l'appellation NTIC s'efface aujourd'hui au profit d'intitulés plus humains comme celui d'«humanités numériques », ou de notions plus sociales comme celle de «transformation digitale », preuve s'il en fallait d'un net besoin de remettre l'homme et ses pratiques au cœur du dispositif, les technologies numériques omniprésentes demeurent néanmoins fondamentalement dominantes dans toutes les réflexions touchant à l'innovation.

Albert Einstein nous l'aurait pourtant bien dit, selon une réflexion qui lui est couramment attribuée, «ce qui caractérise notre époque, c'est la perfection des moyens et la confusion des fins ». Dans le même esprit, on peut dire un peu plus d'un demi-siècle après lui, que ce qui caractérise notre économie moderne, c'est la perfection des technologies et la profusion des outils qui, combinée à la faiblesse des concepts d'emploi associés (la méthodologie), engendre une certaine confusion des fins.

\section{Épistémè et écosystème de l'innovation : un « ordre classique » à retrouver}

Dans un environnement économique que l'on peut se représenter comme un écosystème de l'innovation dont il s'agirait de tirer tous les enseignements afin d'être performant et compétitif, la quintessence de toute problématique à laquelle peuvent être confrontés les acteurs économiques en termes de méthodologie peut se ramener à un problème d'exploitation d'un capital de connaissances qu'il faut acquérir, développer et mettre en valeur avec intelligence. C'est un problème d'intelligence économique au sens premier du mot économie qui suggère une bonne administration de la maison commune. L'économie en effet, au sens que nous indique l'étymologie, c'est la bonne gestion de la maison ${ }^{1}$. L'administration de notre maison commune implantée dans cet écosystème de l'innovation est une discipline qui doit se pratiquer avec méthode en gardant le cap sur une finalité qui, même si elle se matérialise par une production assurément technique, n'en demeure pas moins fondée sur une volonté profondément humaine, traduite par une intention de produire un bien commun. L'appellation « intelligence économique », parfois décriée, souvent incomprise, convient néanmoins assez justement pour désigner cette discipline dont la finalité est de satisfaire l'intérêt général en organisant des mesures performantes (compétitives) d'édification d'un bien commun (notre maison commune).

Pour aborder cette discipline sous l'angle de l'innovation, il faut selon nous envisager un véritable changement de paradigme scientifique en redonnant à l'épistêmê aristotélicienne ${ }^{2}$ toute sa force appuyée sur sa vertu première, l'intelligence (le noûs). La "con-naissance», au sens de l'épistêmê d'Aristote associée au noûs, est la genèse d'une représentation «co-ordonnée » du réel, mise «en forme »

\footnotetext{
1 Le suffixe «-nomie » vient du grec nómos (loi) ou parfois gnômôn (ce qui sert de règle, d'indicateur ou d'instrument de connaissance, soit une référence au nombre dans la tradition pythagoricienne).

2 L'épistêmê chez Aristote, terme que l'on peut traduire sommairement par «connaissance scientifique », réunit théorie (theôría), pratique (praxis) et production (poïsisis), associées chacune à leur vertu respective, sagesse (sophia), méthode (phronêsis) et technique (technè).
} 
(in-formation) par «con-struction » de sens dans une mémoire ${ }^{3}$ organisée, à partir d'une observation (theôría) exécutée avec sagesse (sophia), suivie d'une pratique (praxis) exercée avec méthode (phronêsis), puis d'une production (poïesis) réalisée avec art (technè). La doxa au sens d'Aristote (idée partagée par la plupart parce qu'elle répond à une attente générale des sages), qui n'a pas la connotation péjorative que le discours moderne a retenu à la suite de Platon (cf. plus loin, la notion d'endoxe), vient compléter le dispositif pour refermer la boucle et relancer une observation lorsque la connaissance ainsi construite ne répond pas suffisamment à l'attente. L'observation est ainsi orientée par l'intention, ou une envie partagée par tous ou la plupart, qui fait sens. Elle est guidée par la méthode pour aboutir à la conception de formes nouvelles (design), dans un cycle ininterrompu que l'on peut désigner par l'expression «cycle de l'information ». En évitant cette sorte de précipitation qui incite trop souvent à anticiper sur les solutions et ruine ainsi toute ambition d'innover avec discernement ${ }^{4}$, ce mode de fonctionnement cyclique permet néanmoins d'orienter la pratique, tout en ne se privant pas de nombreuses connaissances utiles à la satisfaction de cette intention collective.

Une telle forme d'innovation serait en totale rupture avec les errements actuels, déjà dénoncés en son temps par Einstein, qui favorisent la confusion entre la fin et les moyens en inversant les rôles entre méthode et technique. Aujourd'hui en effet, le produit (poïesis) qui est technique (technè), est élevé au rang de moyen en lieu et place de la pratique (praxis) qui est méthode (phronêsis), tandis que la finalité qui donne tout son sens à l'action passe à la trappe. La technè élevée au rang de moyen remplace dès lors la méthodologie par une « technologie » déboussolée car privée de cette finalité qui fait la qualité du produit et donne son sens à l'action.

L'élaboration des concepts d'emploi c'est-à-dire la méthodologie au sens noble du terme que l'étymologie doit nous rappeler, soit le discours sur le chemin à suivre pour respecter la vertu de prudence (phronêsis) associée à l'action pratique (praxis), demeure le chaînon manquant des processus d'innovation actuels focalisés sur la technologie. En n'envisageant comme outils de progrès que des artefacts (le couple poïesis/technè), on escamote cette étape essentielle de l'épistêmè aristotélicienne, la pratique méthodique (le couple praxis/phronêsis) qui intègre le concept d'emploi des artefacts dans leur processus de conception. Pour être plus précis ou plus complet, dans les trois couples «activité/vertu associée », qui se succèdent pour constituer l'épistêmè d'Aristote, on ne prête guère attention aux vertus : sophia, sagesse ou discernement, associée à l'observation (theôría), phronêsis, méthode ou prudence, associée à la pratique (praxis), et technè, art ou façon, associée à la production (poïésis). Ces vertus éminemment humaines sont superbement ignorées dans le contexte numérique ou algorithmique qui caractérise nos révolutions technologiques actuelles et emplit de manière quasi monopolistique l'horizon du progrès en s'adossant au concept d'intelligence artificielle.

Les sciences humaines, qui s'interrogent souvent sur les profits à tirer des révolutions technologiques en cours, ne peuvent pas se contenter de ce rôle très théorique au sens premier du terme, qui se limiterait à l'observation des inventions et des prouesses techniques des sciences dites exactes, dans le seul but d'en tirer d'hypothétiques profits sans avoir à en subir les effets néfastes. Privés du soutien d'une intention clairement établie pour leur donner sens, ces résultats ne pourraient en aucun cas prétendre au statut d'innovation à mettre au crédit des sciences humaines. Chercher à innover ainsi, ce serait en effet, anticiper sur les solutions techniques en se privant de l'examen préalable des éléments culturels et cognitifs qui s'imposent à la satisfaction de l'intérêt général.

\footnotetext{
3 Par «mémoire », il faut entendre ici ce dispositif central du système cognitif qui met en œuvre un ensemble de mécanismes permettant l'acquisition et l'encodage de l'information (processus de transformation qui permet de muer les perceptions en éléments ayant du sens), sa rétention (l'enregistrement de ces informations de manière à ce qu'elles puissent être réutilisées plus tard) et sa restitution (récupération des informations préalablement stockées).

4 C'est cette capacité de discernement qui fait toute la singularité de la fonction cognitive opérée par les organismes vivants par rapport à celle susceptible d'être opérée par des artefacts (l'intelligence artificielle). Le mode de fonctionnement cyclique qui permet à nos systèmes cognitifs de ne pas s'encombrer inutilement d'une multitude de données externes, mais de ne retenir que celles répondant à un besoin avéré, est probablement une caractéristique commune aux organismes vivants. Chez l'homme, il est donc naturellement à l'œuvre dans toute la part inconsciente de la fonction cognitive. En ce qui concerne sa part consciente qui se traduit par la pensée en passant par le langage, bien que tout aussi naturel, ce mode itératif peut être parfois négligé, comme ce serait le cas d'un travail d'innovation qui anticiperait sur la solution de problèmes conjoncturels plutôt que de chercher à améliorer ses connaissances en fonction d'un besoin plus général sans cesse affiné.
} 
À l'inverse, le retour à un certain «ordre classique », auquel l'épistêmê d'Aristote nous invite, pourrait faire mentir Michel Foucault qui craignait avec raison de voir évoluer l'épistémè moderne ${ }^{5}$ (la connaissance scientifique de son époque) vers une disparition de l'homme qui «s'effacerait, comme à la limite de la mer un visage de sable » (Foucault, 1966, p. 398). En se laissant porter par les modes technologiques successives, on peut craindre en effet que l'intelligence artificielle, les robots, le big data, l'internet des objets, qui, comme tout ce que l'on associe généralement à la «transformation digitale », ne sont que des outils, finissent par marginaliser la dimension humaine de la décision dans l'action et de la méthode qui la guide. Même s'il ne s'agit pas de contester l'utilité évidente de ces outils, la décision éclairée repose sur cette intelligence (noûs), vertu associée à l'épistêmê d'Aristote, dont le caractère éminemment humain ne peut être ignoré. L'intuition qui enclenche le processus décisionnel, l'intention qui lui donne tout son sens, la volonté qui l'anime, l'émotion qui le stimule et l'attachement à certaines valeurs qui le tempère, sont autant de facultés bien humaines, que l'intelligence artificielle ne pourra jamais reproduire à l'échelle individuelle ou que seuls le big data et les algorithmes associés sont bien incapables d'apporter à la constitution d'une véritable intelligence collective.

\section{Révolution technologique ou transformation digitale ?}

Nous sommes en plein coeur de la quatrième révolution industrielle, une époque charnière de transition entre le XX $X^{e}$ siècle analogique et le XXI siècle numérique (Cavazza, 2019).

En réalité, plutôt que de considérer le phénomène «numérique » comme une quatrième révolution industrielle après la vapeur, l'électricité, puis l'électronique, il semble plus utile de le rapprocher de ces grandes « révolutions culturelles et cognitives » observées par Michel Serres (2007), en le rattachant au phénomène «électronique $»^{6}$ auquel il conviendrait plutôt d'accorder ce statut de troisième « révolution culturelle et cognitive » après l'écriture et l'imprimerie. Son impact sur l'humanité et la civilisation est en effet plus comparable à celui que l'écriture, puis l'imprimerie ont eu chacune à leur tour. Comme ces deux révolutions qui l'ont précédée, elle s'applique avant tout au verbe et à l'information qu'il porte, en bouleversant tout sur son passage (société, politique, économie...). Comme les deux premières, elle est ainsi fondamentalement analogique plutôt que numérique, au même titre que la langue, support incontournable de ces trois révolutions dont elle est le ciment commun.

Michel Serres nous explique en effet qu'il n'y a pas d'objet « dont nous ne puissions pas dire qu'il stocke, traite, émet et reçoit de l'information ». Cette « quadruple caractéristique commune à tous les

\footnotetext{
5 Après «la Renaissance », Michel Foucault constate « deux grandes discontinuités dans la culture occidentale : celle qui inaugure l'âge classique (vers le milieu du XVII ${ }^{\mathrm{e}}$ siècle) et celle qui, au début du XIX ${ }^{\mathrm{e}}$ siècle, marque le seuil de notre modernité » (Foucault, 1966, p.13). Dans l'épistémè classique, ce sont l'ordre, l'identité et la différence qui servent à forger la représentation du Monde. «La vocation du langage classique a toujours été de faire tableau : que ce soit le discours naturel, recueil de la vérité, description des choses, corpus de connaissances exactes, ou dictionnaire encyclopédique » (ibid., p. 332). C'est le temps essentiel de la conception qui fait de la pratique une méthode et de la période classique un âge de raison. C'est l'époque de la Grammaire générale et raisonnée contenant les fondemens de l'art de parler, expliqués d'une manière claire et naturelle (Arnauld \& Lancelot, 1660) ou de la Logique de Port-Royal (Arnauld \& Nicole, 1668), proposant un idéal de langage rationnel qui voulait concilier esprit de finesse et esprit de géométrie. C'est en fin de période, l'époque de l'Encyclopédie de Paris ou Dictionnaire raisonné des sciences, des arts et des métiers (Diderot \& d'Alembert, 1751), puis d'Yverdon ou Dictionnaire universel raisonné des connaissances humaines (de Felice, 1771). C'est aussi en France, l'époque de Richelieu, de la centralisation monarchique, des Académies et d'une esthétique inspirée par un idéal d'équilibre, d'harmonie, de simplicité, d'unité, de rationalité, de discipline, de stabilité et d'humilité (l'artiste disparaît dans son œuvre), tandis qu'en Italie, berceau de la Renaissance, l'art baroque fait son apparition, caractérisé à l'opposé par la démesure des formes stimulée par des techniques en pleine expansion. De la même manière qu'en Italie, les Beaux-arts s'épanouissent aux $\mathrm{XVII}^{\mathrm{e}}$ et XVIII ${ }^{\mathrm{e}}$ siècles dans le style baroque, l'extraordinaire développement des techniques en Amérique du Nord et en Europe aux $\mathrm{XIX}^{\mathrm{e}}$ et $\mathrm{XX}^{\mathrm{e}}$ siècles, et en particulier le formidable impact de l'informatique et de la numérisation de l'information, se traduisent par une profusion d'innovations et de pratiques nouvelles. Face à un tel foisonnement de la science pratique aiguillonnée par des techniques en perpétuelle ébullition, sans véritable conscience méthodologique reposant sur des bases théoriques solides incluant une recherche permanente (philo) de sagesse (sophia), seul un travail de mise en ordre et de méthode paraît apte à relever avec prudence (phronêsis) le défi du modernisme.

6 Par «phénomène électronique », il faut entendre la manifestation des retombées d'un ensemble de nouvelles technologies liées à l'électronique, allant jusqu'à la nanoélectronique en passant par la microélectronique (là où l'électronique classique allait dans le sens d'une miniaturisation toujours croissante donnant naissance à la microélectronique, la nanoélectronique procède à l'inverse en allant du plus petit, l'atome et ses électrons, vers le plus grand pour créer des objets manufacturés).
} 
objets du monde », qu'ils soient « vivants » ou «inertes », est le résultat d'un " couplage entre un support et un message » (Serres, 2007, pp. 127 et 128). Chez l'homme, ce couplage avait pour support le corps, la mémoire et la voix qui assuraient le stockage, le traitement et l'émission au moyen de la langue qui est analogique. Avec l'invention de l'écriture, puis plus tard, celle de l'imprimerie et aujourd'hui, celle de l'ordinateur, c'est ce couplage qui s'externalise en s'affranchissant du corps grâce à la peau de bête, au papyrus ou au papier, puis à l'imprimé et enfin au support numérique. Chacune de ces révolutions induit son lot de bouleversements politiques, économiques, scientifiques, religieux et éducatifs : inventions de l'État, de la monnaie, de la géométrie, des grands monothéismes et de la pédagogie avec l'écriture, naissance du capitalisme, de la science expérimentale, du mouvement de la Réforme et de la démocratie moderne avec l'imprimerie.

Le support numérique est en train de bouleverser notre civilisation comme celui de l'écriture puis de l'imprimerie l'ont fait chacun en leur temps, peut-être même encore bien plus profondément que les deux précédents n'ont pu le faire. Cette véritable «révolution» est bien avant tout «culturelle et cognitive ». La technologie n'en est que le facteur déclenchant que l'on peut considérer comme un marqueur, mais sûrement pas comme l'élément porteur. C'est l'économie, au sens étymologique du terme, la bonne gestion de notre maison commune, qui en est le vecteur. L'intelligence humaine ou la raison (le noûs), vertu associée à l'épistêmê et indissociable de la notion de discernement que l'on retrouve dans celle de sens ${ }^{7}$, doit nous aider à guider cette économie de la connaissance dans l'épais brouillard du progrès en marche.

Autrement dit, et pour résumer ce qui précède, l'innovation doit être au cœur de nos préoccupations méthodologiques en matière d'économie. L'intérêt général ${ }^{8}$, dont on doit se demander comment le satisfaire, est la finalité qui s'applique à la gestion d'un bien commun (l'économie). Le contexte de révolution culturelle et cognitive, dont l'électronique et le numérique ne sont que les marqueurs technologiques, se répercute sur l'ensemble du spectre scientifique et par là, sur l'ensemble du fonctionnement de nos sociétés humaines. L'intelligence économique doit être en première ligne pour contribuer à anticiper cet impact avec discernement afin que cette révolution se réalise en préservant l'intérêt général. Cette discipline, qui met l'information et l'anticipation par l'innovation, au service de la performance et de la compétitivité, et dont on a vu que la finalité était de satisfaire l'intérêt général pour l'édification d'un bien commun (notre maison commune), impacte ainsi ce que certains auteurs nomment «intelligence collective » (la performance par l'innovation) et «intelligence sociale » (la performance par la transformation) ${ }^{9}$.

Information et innovation sont les deux piliers de cette «transformation digitale » caractéristique de la révolution culturelle et cognitive en cours qui impacte profondément la gestion de notre maison commune (l'économie).

\section{LA PENSEE SYSTEMIQUE ET L'INTELLIGENCE COLLECTIVE AU CHEVET DE LA TRANSFORMATION DIGITALE}

L'intelligence économique peut être ce projecteur qui éclaire l'administration de notre maison commune pour anticiper les nombreux impacts sociaux de la révolution électronique en cours. Elle s'appuie sur une intelligence collective qui repose sur l'élaboration d'une pensée collégiale. Cinq à six mille ans après l'invention de l'écriture et cinq à six cents ans après celle de l'imprimerie, l'électronique qui est une branche de la physique appliquée utilisant l'électricité comme support pour le traitement, la transmission et le stockage d'informations, prolonge les deux premières révolutions en nous donnant les

\footnotetext{
7 «Puissance innée de discernement » chez Aristote (Pelletier, 2007, p. 33).

8 Si on s'interroge sur le périmètre qu'il faut donner à l'intérêt général, on peut utilement méditer cette pensée de Montesquieu : «Si je savais quelque chose qui me fût utile et qui fût préjudiciable à ma famille, je la rejetterais de mon esprit. Si je savais quelque chose d'utile à ma famille et qui ne le fût pas à ma patrie, je chercherais à l'oublier. Si je savais quelque chose utile à ma patrie et qui fût préjudiciable à l'Europe, ou bien qui fût utile à l'Europe et préjudiciable au genre humain, je la regarderais comme un crime » (Pensées, $\left.\mathrm{n}^{\circ} 741\right)$.

9 (Chantaraud, 2019).
} 
moyens d'une intelligence collective. Cette intelligence collective, élément moteur d'une pensée systémique adaptée à la complexité des grandes transitions, doit être à la fois l'aboutissement et l'instrument de la transformation digitale qu'elle éclaire.

\section{Mémoire collective et langage documentaire : l'intelligence collective, instrument de transformation}

De même que l'intelligence et la pensée individuelle reposent sur la mémoire déclarative et un langage qui l'organise, l'intelligence et la pensée collective reposent sur une mémoire collective et un langage commun qui l'organise. Cette mémoire et ce langage sont documentaires. Mémoire collective et langage commun sont en effet fortement touchés par les évolutions du document support du discours, trait commun de ces trois révolutions culturelles et cognitives identifiées par Michel Serres, et élément clé de cette intelligence sociale à laquelle la transformation digitale nous invite.

À ce stade de la réflexion, la question qui doit se poser à nous est, selon moi : « comment faire pour que l'intelligence économique soit à la hauteur de cet enjeu de transformation digitale à l'origine de la révolution culturelle et cognitive en cours ?». Pour y répondre, je propose d'aborder l'exploitation du capital de connaissances que nous avons pour ambition de développer (1'intelligence collective) et de mettre en valeur (l'intelligence sociale), en adoptant une démarche que l'on pourrait qualifier d'épistémique au sens d'Aristote, et en appliquant à la pensée collective cette réflexion très aristotélicienne de Michel Volle ${ }^{10}$ qui mérite d'être largement méditée :

«Notre pensée est essentiellement pratique, orientée vers l'action : la grille conceptuelle à travers laquelle elle perçoit un objet est déterminée par la situation qui nous met en relation avec lui et par notre intention envers lui. En regard de la complexité illimitée de l'objet, cette pensée est simple »(Volle, 2017).

Une telle démarche permet de concevoir une pensée collective qui perçoit son objet (l'économie et ses objets ou toute autre sorte d'objet suscitant l'intérêt d'une communauté) au travers d'une grille conceptuelle déterminée par la situation qui nous met en relation avec ces objets, et par notre intention envers eux émanant de nos attentes (l'intérêt général). Cette pensée doit être essentiellement pratique, orientée vers l'action. En regard de la complexité illimitée de son objet, elle peut être relativement simple.

La grille conceptuelle à travers laquelle cette pensée collective va percevoir notre maison commune, en relation avec une situation en perpétuelle évolution dont nous souhaitons tirer profit dans l'intention de satisfaire l'intérêt général, va reposer sur un langage commun, dont l'usage doit donc être simple. En regard de la complexité illimitée de l'objet « économie », son usage peut être aussi simple en effet que celui d'une langue maternelle, dont un enfant de trois ans arrive peu à peu à maîtriser les rudiments, malgré la complexité du monde autour de lui qui est l'objet de toutes ses pensées. Comme n'importe quelle langue, ce langage commun repose sur une grammaire dont mon travail de thèse détaille le concept (Beau, 2019) et que je peux tenter de résumer ici en quelques lignes.

\section{Grammaire commune et grille conceptuelle pour l'élaboration d'une pensée collective}

Notre grille conceptuelle s'articule autour de deux axes : en abscisse, une sémantique horizontale qui cible des catégories déterminées par l'intention émanant de l'attente fonctionnelle ${ }^{11}$, et en ordonnée une syntaxe verticale qui décline leurs attributs déterminés par la situation (cf. plus loin 2). Cette grammaire

\footnotetext{
${ }^{10}$ Polytechnicien, docteur en histoire économique, administrateur de l'INSEE et spécialiste des systèmes d'information, Michel Volle a contribué à l'organisation de la maîtrise d'ouvrage du système d'information de plusieurs entreprises (Air France, ANPE...). Il a participé en 1997 à la création du Club des maîtres d'ouvrage des systèmes d'information dont il est président d'honneur (source Wikipédia).

${ }^{11}$ Fonction économique ou toute autre fonction collective.
} 
permet ainsi d'élaborer une pensée collective dans une mémoire documentaire opérant une capitalisation des connaissances réunies par une communauté définie autour de sa fonction, source d'intérêt général ${ }^{12}$.

> Dans la démarche épistémique adoptée, la connaissance (épistêmê) est considérée comme une composition reposant sur la dimension conceptuelle de notre système cognitif : la raison (ou intelligence) que l'on peut associer à la question « pourquoi ?».

C'est la réponse à cette question «pourquoi » qui indique le cap à suivre. C'est elle qui, tout au long du travail des acteurs économiques, doit donner tout son sens à la démarche d'exploitation d'une mémoire collective consacrée à une gestion intelligente de la maison commune (l'économie). Elle correspond à une « attente des sages », la doxa aristotélicienne ou l'endoxe ${ }^{13}$ (cf. précédemment, la notion de boucle), qui fait de ce capital de connaissances à mettre en commun et qu'il convient de développer et de mettre en valeur, une mémoire collective dynamique en perpétuelle révolution (cycle de l'information). Celleci pourra en effet s'enrichir ainsi en permanence de nouvelles connaissances issues de multiples expériences et de cette ardente volonté novatrice qui doit animer toute activité économique.

La mise en œuvre d'une telle mémoire collective nécessite un minimum d'organisation qu'un référentiel documentaire conçu comme un langage commun parlé par tous, est en mesure d'apporter. Cette organisation peut s'inspirer de la démarche aristotélicienne, qui distingue trois étapes dans la construction de l'épistêmê. Celles-ci sont adossées dans notre mémoire à notre système sensoriel alimentant la dimension conceptuelle de notre système cognitif qui, nous l'avons vu, donne son sens à l'ensemble, en réponse à la question "pourquoi ? ». Ces trois étapes épistémiques associées à nos sens se déclinent à leur tour, en réponse à six grandes questions ontologiques.

Tout commence par l'observation (theôría) de l'environnement, reposant sur la dimension spatiale de notre système sensoriel : la vue (coup d'œil, vision), le toucher (tact, contact) et le goût (discernement, appréciation) que l'on peut associer respectivement aux questions « quoi ? », « où ? » et « comment ? ».

$>$ L'observation spatiale cède ensuite la place à l'action, la pratique (praxis) reposant sur la dimension temporelle de notre système sensoriel : l'ouïe (entendement, entente) et l'odorat (flair, sentiment) que l'on peut associer aux questions « quand? » et « qui ? ».

$>$ Puis, l'ensemble débouche sur un produit (poïesis) reposant sur une dimension factuelle de notre système sensoriel : l'intuition (pensée, jugement), ce sixième sens qui fait la synthèse des cinq précédents, que l'on peut associer à la question «combien ? » au sens premier du terme (comment bien) qui délivre un jugement pouvant être aussi bien qualitatif que quantitatif.

Les cinq sens complétés par l'intuition sont ainsi gouvernés, dans notre système cognitif, par la raison qui fait sens à partir d'une attente, selon une hiérarchie des sens récapitulée dans la table 1 qui suit, dont on peut s'inspirer pour la conception d'une mémoire collective et le langage documentaire commun associé.

\footnotetext{
${ }^{12} \mathrm{La}$ «transformation digitale » peut être envisagée comme une source d'intérêt général que la gestion de notre bien commun (la fonction économique) doit permettre de satisfaire en toute intelligence.

${ }^{13}$ Néologisme utilisé par certains exégètes d'Aristote pour traduire le mot doxa. Le terme «endoxe » (qui va dans le sens de l'attente générale des sages) est conçu par opposition avec la notion de «paradoxe » (Pelletier, 2007, p. 43), qui va contre l'attente générale des sages. L'usage de ce néologisme permet de se démarquer du sens péjoratif issu de la tradition platonicienne dont Aristote se distingue, mais que notre époque moderne a retenu en assimilant la doxa à une opinion reçue ou à un préjugé, voire à un dogme intangible.
} 
Table 1. La hiérarchie des sens : de l'observation oculaire à la composition intelligente.

\begin{tabular}{|c|c|c|c|c|c|}
\hline dimension cognitive & activité associée & sens impliqué & besoin associé & moyen d'action & matière cognitive \\
\hline & & vue (coup d'œil) & quoi & \multirow{6}{*}{ numérique (algorithmes) } & \multirow{6}{*}{ données } \\
\hline spatiale & observation (theôría) & toucher (tact) & où & & \\
\hline & & goût (discernement) & comment & & \\
\hline \multirow{2}{*}{ temporelle } & \multirow{2}{*}{ pratique (praxis) } & ouïe (entendement) & quand & & \\
\hline & & odorat (flair) & qui & & \\
\hline factuelle & production (poïésis) & intuition (jugement) & combien & & \\
\hline conceptuelle & composition & raison (intelligence) & pourquoi & analogique (pensée) & connaissance \\
\hline
\end{tabular}

\section{Le concept de plan d'indexation et l'index analogique}

L'indexation n'est pas seulement envisagée ici comme un but en soi pour faciliter l'accès d'usagers divers et variés à la connaissance ou aux documents qui la portent, mais plus directement comme un moyen d'anticiper et de satisfaire les besoins de savoir d'une communauté en organisant sa recherche d'information. Elle procède, en amont, à l'analyse du besoin de savoir de la communauté réunie autour d'une activité déterminée, puis à sa traduction dans les termes d'un plan d'action à l'usage de la dite communauté pour satisfaire ce besoin. Elle sera dite « analogique $»^{14}$ pour indiquer la nature du rapport entre l'information retenue et l'indice de classification qui lui est attribué. Notre index analogique est donc un plan (ou plan d'indexation) des sujets traités ou à traiter par le système d'information documentaire de la communauté, qui associe des indices de classification à des intitulés avec lesquels les contenus des documents ou des parties de document indexés (l'information retenue ou à retenir) entretiennent un rapport de ressemblance.

La communauté de fonction réunie autour de ce langage commun, peut ainsi planifier son activité documentaire à travers différents plans d'indexation qui s'organisent dans une hiérarchie (syntaxe verticale) en titres, chapitres, dossiers, puis documents sources, de travail et produits, déterminés par la situation et ciblant à chaque niveau hiérarchique six objets distincts (sémantique horizontale) déterminés par l'intention émanant de l'attente fonctionnelle. Ces plans permettent à la dite communauté de coordonner sa veille (titres, chapitres, dossiers) et d'orchestrer sa production (documents sources, documents de travail, production) en se calquant sur le modèle fourni par l'organisation de nos sens pour répondre aux six questions (quoi, où, comment, quand, qui, combien) gouvernées par le pourquoi de la raison. À partir de là, on peut proposer une organisation de la mémoire documentaire conçue comme une grille conceptuelle (cf. Table 2), pour recueillir les connaissances issues de multiples perceptions ou expertises différentes dans des plans de dossiers indexant leurs supports documentaires. Chaque plan est ainsi conçu comme une mémoire documentaire collective organisée selon une grammaire commune dont la table 2 ci-après donne le principe : la grille conceptuelle fonctionnelle à travers laquelle la communauté perçoit les objets avec lesquels la situation la met en relation, et sur lesquels elle a conçu l'intention de développer une pensée collective, est essentiellement pratique, orientée vers l'action que la fonction commande.

\footnotetext{
${ }^{14}$ Voir à ce sujet, « Le principe d'indexation analogique » (Beau, 2019, pp. 295-302).
} 
Table 2. Grille conceptuelle fonctionnelle (le principe d'indexation documentaire).

\begin{tabular}{|c|c|c|c|c|c|c|c|c|}
\hline \multirow{2}{*}{\multicolumn{3}{|c|}{$\begin{array}{l}\text { SYNTAXE (situation) } \\
\text { indices i, j, k, l, m, n }\end{array}$}} & \multicolumn{6}{|c|}{ SÉMANTIQUE (intention ou attente fonctionnelle) } \\
\hline & & & $=\begin{array}{c}0 \\
\begin{array}{c}\text { (panorama }) \\
\text { quoi }\end{array}\end{array}$ & $\begin{array}{l}1 \\
\text { (contexte) } \\
\text { où }\end{array}$ & $\begin{array}{l}2 \\
\text { (propriété) } \\
\text { comment }\end{array}$ & $\begin{array}{l}3 \\
\text { (dynamique) } \\
\text { quand }\end{array}$ & $\begin{array}{c}4 \\
\text { (protagonistes) } \\
\text { qui }\end{array}$ & $\begin{array}{c}5 \\
\text { (ressource) } \\
\text { combien }\end{array}$ \\
\hline $\begin{array}{l}\text { TITRES } \\
\text { (images) }\end{array}$ & $\mathrm{i}$ & quoi & Lieu & Milieu & Appareil* & Activité & Acteurs & Moyens \\
\hline $\begin{array}{l}\text { CHAPITRES } \\
\text { (formes) }\end{array}$ & $\mathrm{ij}$ & où & Mode générique & Mode contextuel & Mode fonctionnel & Mode agile & Modes singuliers & Mode critique \\
\hline $\begin{array}{l}\text { DOSSIERS } \\
\text { (teneurs) }\end{array}$ & $\mathrm{ijk}$ & comment & $\begin{array}{l}\text { Contenu } \\
\text { synoptique }\end{array}$ & $\begin{array}{l}\text { Contenu } \\
\text { relationnel }\end{array}$ & Contenu organique & $\begin{array}{c}\text { Contenu } \\
\text { opérationnel }\end{array}$ & $\begin{array}{l}\text { Contenus } \\
\text { humains }\end{array}$ & $\begin{array}{c}\text { Contenu } \\
\text { névralgique }\end{array}$ \\
\hline $\begin{array}{l}\text { SOURCES } \\
\text { (discours) }\end{array}$ & ijkl & quand & $\begin{array}{l}\text { Documentation de } \\
\text { synthèse }\end{array}$ & $\begin{array}{l}\text { Documentation } \\
\text { généraliste }\end{array}$ & $\begin{array}{l}\text { Documentation } \\
\text { institutionnelle }\end{array}$ & Médias & $\begin{array}{l}\text { Documentation } \\
\text { scientifique }\end{array}$ & $\begin{array}{l}\text { Documentation } \\
\text { sensible }\end{array}$ \\
\hline $\begin{array}{l}\text { ANALYSES } \\
\text { (traces) }\end{array}$ & ijklm & qui & Suivi d'ensemble & Examen formel & Étude de fond & Veille & Enquêtes & Évaluation \\
\hline $\begin{array}{l}\text { PRODUITS } \\
\text { (idées) }\end{array}$ & ijklmn & combien & Notes de lecture & Fiches & $\begin{array}{l}\text { Monographies, } \\
\text { mémoires }\end{array}$ & $\begin{array}{l}\text { Bulletins } \\
\text { périodiques }\end{array}$ & $\begin{array}{l}\text { Courriers } \\
\text { personnalisés }\end{array}$ & Essais \\
\hline
\end{tabular}

L'économie en général ou chacun de ses objets, en l'occurrence, tous les objets dont la maîtrise (récolte, conception, traitement, gestion, production, commercialisation...) relève de l'économie, que nous regrouperons sous le terme générique de "produits », peuvent ainsi, en utilisant ce modèle, faire l'objet d'un plan d'indexation ou Plan des Dossiers d'Intelligence Économique (PDIE). En s'inspirant de la stratégie militaire ${ }^{15}$ que le renseignement de défense permet d'éclairer ${ }^{16}$, la transformation digitale que l'intelligence économique se propose d'éclairer, peut se définir comme une fonction de compétitivité (intention) ou un art de la dialectique des volontés destiné à atteindre des objectifs fixés par le marché ${ }^{17}$, en utilisant au mieux les moyens dont on dispose.

\section{De la sécurité au bien commun, en passant par la compétitivité et le progrès, l'exemple militaire : l'intelligence collective, aboutissement de la transformation}

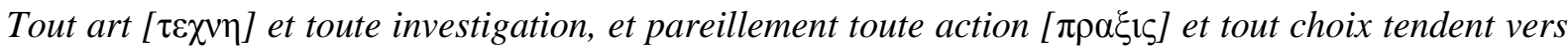

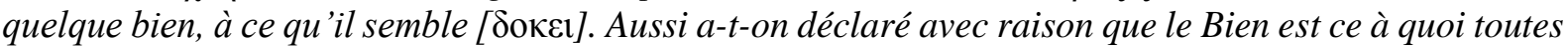
choses tendent (Aristote, trad. 1959).

L'exemple militaire du renseignement de défense peut s'appliquer à d'autres domaines que celui de la sécurité ou de la compétitivité pour aboutir au bien commun, en passant par un progrès dont la maîtrise relève d'une éthique que nous inspire l'enseignement d'Aristote. Un tableau de correspondance des concepts utiles à l'indexation de dossiers dans différents plans à vocation stratégique, économique, scientifique et politique (cf. Table 3) peut être établi à partir de l'exemple du renseignement de défense ${ }^{18}$. De la stratégie militaire à la politique publique, la modélisation systémique de notre monde du XXI ${ }^{\mathrm{e}}$ siècle passe en effet par une économie de marché en pleine transformation digitale associée

\footnotetext{
${ }^{15}$ Mode d'action ou « art de la dialectique des volontés » destiné à « atteindre les objectifs fixés par la politique en utilisant au mieux les moyens dont on dispose » selon le général Beaufre (1962).

${ }^{16}$ La défense en général ou chacun de ses objets, en l'occurrence, tous les objets dont la maîtrise (détection, analyse, traitement, négociation, protection, combat...) relève de la défense, que nous regroupons sous le terme générique de "menaces », peuvent faire l'objet d'un Plan des Dossiers du Renseignement de Défense (PDRD).

${ }^{17}$ Le marché est entendu ici au sens d'Adam Smith comme un ensemble de lois naturelles et de conventions sociales organisant la production de biens marchands en établissant leur valeur par confrontation de l'offre et de la demande. Il peut s'assimiler à un processus de validation sociale de la production des biens marchands, comme la politique peut être considérée comme un processus de validation sociale du gouvernement des hommes.

${ }^{18}$ Cf. (Beau, 2019).
} 
à une science que l'on voudrait universelle. Cette dernière serait caractérisée par une épistémè que l'on dira « organique » pour faire référence à l'Organon développant la Logique d'Aristote, et qualifier cette épistémè aristotélicienne dont nous avons tenté de défendre le prolongement dans la perspective tracée par Michel Foucault. La stratégie militaire, qu'une revue stratégique permanente doit adapter à la transformation digitale en cours profondément ancrée dans notre économie de marché, peut nous servir ainsi de modèle. L'éthique fondée sur une véritable épistémè organique, gage d'une science à vocation universelle, peut alors nous guider pour concevoir un nouveau paradigme politique sur lequel toute politique publique tendant à la recherche du Bien commun doit pouvoir s'appuyer.

Table 3. Correspondance des concepts utiles à l'indexation des dossiers dans les différents plans.

\begin{tabular}{|c|c|c|c|}
\hline $\begin{array}{c}\text { revue stratégique } \\
\text { STRATÉGIE MILITAIRE } \\
\text { fonction stratégique } \\
\text { " connaissance et anticipation » } \\
\text { (renseignement de défense) }\end{array}$ & $\begin{array}{l}\text { transformation digitale } \\
\text { ÉCONOMIE DE MARCHÉ } \\
\text { fonction économique } \\
\text { « information et innovation » } \\
\text { (intelligence économique) }\end{array}$ & $\begin{array}{l}\text { épistémè organique } \\
\text { SCIENCE UNIVERSELLE } \\
\text { fonction scientifique } \\
\text { « savoir et prédiction » } \\
\text { (recherche scientifique) }\end{array}$ & $\begin{array}{l}\text { paradigme politique } \\
\text { POLITIQUE PUBLIQUE } \\
\text { fonction politique } \\
\text { " réflexion et perspective " } \\
\text { (intelligence collective) }\end{array}$ \\
\hline \multicolumn{4}{|c|}{ DOMAINES associés } \\
\hline prévention & prospective & prédiction & prévoyance \\
\hline dissuasion nucléaire & dystopie cybernétique* & paradigme positiviste ${ }^{* *}$ & révolution \\
\hline protection & veille & précaution & vigilance \\
\hline intervention & influence & pratique & action \\
\hline \multicolumn{4}{|c|}{ INTENTIONS } \\
\hline Sécurité & Compétitivité & Progrès & Bien \\
\hline \multicolumn{4}{|c|}{ SITUATIONS (angles d'approche ou contextes) } \\
\hline géopolitique & méthodologie & épistémologie & idéologie \\
\hline \multicolumn{4}{|c|}{ SUJETS des plans de dossiers (OBJETS d'intérêt) } \\
\hline menaces & produits & choses & défis \\
\hline \multicolumn{4}{|c|}{ GOUVERNANCES } \\
\hline politique & marché & discipline & éthique \\
\hline \multicolumn{4}{|c|}{ LIEUX fonctionnels (champs d'action) } \\
\hline territoire & champ d'activités & champ conceptuel & communauté \\
\hline \multicolumn{4}{|c|}{ INSTRUMENTS « dialectiques » } \\
\hline force & performance & discours & administration \\
\hline \multicolumn{4}{|c|}{ MOYENS techniques } \\
\hline armement & outil numérique & instrumentation & technologie \\
\hline \multicolumn{4}{|c|}{ MILIEUX fonctionnels (domaines d'action) } \\
\hline Terre & Création & Technique & Nation \\
\hline Mer & Information & Théorie & Monde cosmopolite \\
\hline Air & Communication & Pratique & Cybermonde \\
\hline
\end{tabular}


La table 3 ci-dessus présente une vue d'ensemble des concepts utiles à l'élaboration de plans d'indexation à vocation politique pour l'intelligence collective (4 $4^{\text {eme }}$ colonne), en s'inspirant d'un modèle réalisé pour le renseignement de défense (1 ${ }^{\text {ère }}$ colonne), adapté à l'intelligence économique ( $2^{\text {ème }}$ colonne), comme à la recherche scientifique ( $3^{\text {ème }}$ colonne). La politique, comme la défense (ou encore l'économie, nous l'avons vu, et la science) ou chacun des sujets susceptibles d'être objet d'intérêt politique (stratégique, économique ou scientifique), c'est-à-dire tous les sujets dont la maîtrise ${ }^{19}$ relève de la politique (de la stratégie, de l'économie ou de la science), que nous regroupons sous le terme générique de «défis » (respectivement «menaces », «produits » ou « choses »), peuvent ainsi faire l'objet de Plans des Dossiers d'Intelligence Collective, du Renseignement de Défense, d'Intelligence Économique ou de la Recherche Scientifique (PDIC, PDRD, PDIE ou PDRS).

La politique ${ }^{20}$ publique, que l'intelligence collective se propose d'éclairer comme le renseignement de défense éclaire la stratégie militaire (ou comme l'intelligence économique peut éclairer l'économie de marché, et la recherche scientifique, la science universelle), est au collectif (ou encore à l'économique et au scientifique) ${ }^{21}$, ce que la stratégie militaire (ou encore l'économie de marché et la science universelle) est à la défense (à l'économique ou au scientifique), un mode d'action, nous l'avons vu, ou un art de la dialectique des volontés destiné à atteindre les objectifs fixés ${ }^{22}$ en utilisant au mieux les moyens dont on dispose.

La fonction politique «réflexion et perspective» ou intelligence collective, est alors au Bien commun (l'intention), ce que la fonction stratégique «connaissance et anticipation » ou renseignement de défense est à la sécurité (ce que la fonction de transformation «information et innovation »ou intelligence économique, est à la compétitivité, et ce que la fonction épistémique « savoir et prédiction » ou recherche scientifique est au progrès), une fonction essentielle permettant d'éclairer tous les domaines en lien avec la dite fonction (politique, de transformation, épistémique ou stratégique) :

- la prévoyance qui est à l'éthique, ce que la prévention est à la politique sécuritaire (ce que la prospective est au marché, ou la prédiction à la discipline scientifique), un moyen d'atteindre ses objectifs anticipant l'avenir ;

- la révolution qui est à l'éthique, ce que la dissuasion nucléaire est à la politique sécuritaire (ce que la dystopie cybernétique est au marché, ou le paradigme positiviste ${ }^{23}$ à la discipline scientifique), un moyen radical d'atteindre ses objectifs ;

- la vigilance qui est à l'éthique, ce que la protection est à la politique sécuritaire (ce que la veille est au marché, ou la précaution à la discipline scientifique), un moyen passif d'atteindre ses objectifs ;

- l'action politique qui est à l'éthique, ce que l'intervention est à la politique sécuritaire (ce que l'influence est au marché, ou la pratique à la discipline scientifique), un moyen actif d'atteindre ses objectifs.

L'expression « dystopie cybernétique » désigne un développement paroxystique de la robotique qui serait associée à l'intelligence artificielle, dans un espace cybernétique impliquant d'autres champs

\footnotetext{
${ }^{19}$ Respectivement : - détection, analyse, traitement, négociation, protection, combat... ; - récolte, conception, traitement, gestion, production, commercialisation... ; - observation, interprétation, compréhension, application... ; - perception, mémorisation, ordonnancement, mise en perspective, diffusion...

${ }^{20}$ «Art, manière de diriger, en vue du bien commun, toutes les activités d'une société » (Dictionnaire de l'Académie française).

${ }^{21}$ Le collectif, l'économique ou le scientifique, désignent ici l'ensemble des phénomènes se rapportant respectivement à la collectivité, à l'économie ou à la science, comme le politique peut servir à désigner l'ensemble des phénomènes se rapportant à la politique.

${ }^{22}$ Par l'éthique politique comme par la politique sécuritaire (ou encore le marché économique et la discipline scientifique). L'éthique peut ainsi être considérée comme un système de conduite qui détermine les objectifs à atteindre en matière de politique publique, comme la politique sécuritaire est un système d'organisation de la cité qui fixe les objectifs à atteindre en matière de stratégie militaire (ou encore comme le marché économique, système d'organisation de la production, détermine les objectifs en matière de transformation digitale, et comme la discipline scientifique, système d'organisation du savoir, fixe les objectifs en matière d'épistémè organique).

${ }^{23}$ Voir un peu plus loin, ce que désignent ces expressions.
} 
scientifiques tels que la biologie ou l'économie par exemple. Comme la dissuasion avec les objectifs de la politique de défense et l'emploi des armes de destruction massive, c'est un moyen radical d'atteindre les objectifs du marché, qui repose sur la menace d'emploi de techniques de production massive.

L'expression «paradigme positiviste » désigne quant-à-elle une approche théorique dominante qui « durcirait » le champ scientifique, au sens où l'on parle de sciences « dures ». Ce serait une mobilisation du discours scientifique s'inscrivant dans une dynamique de progrès débridée, associée à un positivisme systématisé que l'on pourrait qualifier d' «hyperscientifique ». Celui-ci s'imposerait à l'ensemble des disciplines scientifiques, au point d'impacter les humanités en assujettissant tout progrès scientifique aux nouvelles technologies, et allant jusqu'à organiser la convergence des NBIC (Nanotechnologies, Biotechnologies, Informatique et sciences Cognitives) vers ce qu'il est convenu d'appeler une « singularité technologique », concept proche de la « dystopie cybernétique » évoquée plus haut. De tels aboutissements économiques et scientifiques, dont le spectre funeste pèserait lourdement sur notre humanité, ne peuvent être agités pour le moment, fort heureusement, que comme de pures idées exprimées, souhaitons-le, à titre exclusivement dissuasif. On peut reprendre ici la formule consacrée aux fictions: "toute ressemblance avec des situations existantes ou ayant existé ne saurait être que fortuite $» \ldots$ ou véritablement tragique.

L'éthique est ainsi à la politique publique, ce que la politique est à la stratégie militaire (ou ce que le marché est à l'économie de marché ou la discipline à la science universelle), une forme de gouvernance qui lui fixe les objectifs à atteindre. L'idéologie est alors à la politique publique, ce que la géopolitique est à la stratégie militaire (ou ce que la méthodologie est à la transformation digitale, et l'épistémologie à l'épistémè organique), un angle d'approche privilégié pour la détermination des sujets (défis, menaces, produits ou choses), susceptibles de faire l'objet d'un plan d'indexation documentaire à usage collectif (PDIC, PDRD, PDIE ou PDRS).

La communauté est à la fonction politique, ce que le territoire est à la fonction stratégique (et ce que le champ d'activités est à la fonction économique ou le champ conceptuel à la fonction scientifique), un lieu respectivement idéologique ou géopolitique (ou encore épistémologique et méthodologique) défini et construit avec précision, auquel s'applique la fonction (politique, stratégique, économique ou scientifique).

L'administration est à la politique publique, ce que la force est à la stratégie militaire (ou ce que la performance est à l'économie de marché et le discours à la science universelle), l'instrument d'un art de la dialectique employé pour atteindre les objectifs fixés par l'éthique d'une part, et par la politique sécuritaire d'autre part (ou respectivement par le marché économique et la discipline scientifique).

La technologie est à la politique publique, ce que l'armement est à la stratégie militaire (ou ce que l'outil numérique est à l'économie de marché et l'instrumentation à la science universelle), un ensemble de moyens techniques employés pour atteindre les résultats fixés.

Enfin, le cybermonde, est à la fonction politique, ce que l'air (la communication ou la pratique) est à la fonction stratégique (économique ou scientifíque), un « domaine d'action » (ou milieu) dont la maîtrise est source d'agilité et implique une forme dédiée (mode agile) d'instrument de la dialectique des volontés (administration, force, performance ou discours). Le monde cosmopolite ensuite, est à la fonction politique, ce que la mer (l'information ou la théorie) est à la fonction stratégique (économique ou scientifique), un «domaine d'action » (ou milieu) dont la maîtrise démultiplie la portée fonctionnelle (respectivement politique et stratégique, économique ou scientifique) et implique une forme dédiée (mode fonctionnel) d'instrument de la dialectique des volontés. La nation enfin, est à la fonction politique, ce que la terre (la création ou la technique) est à la fonction stratégique (économique ou scientifique), un «domaine d'action» (ou milieu) dont la maîtrise s'inscrit exactement dans le contexte (national, territorial, créatif ou technique) de la fonction et implique une forme dédiée (mode contextuel) d'instrument de la dialectique des volontés.

Imaginons que l'on veuille réunir une communauté d'experts, aux compétences différentes mais complémentaires, pour travailler collectivement sur un sujet très général comme par exemple l'économie de marché, la science universelle ou la politique publique, dans une perspective 
clairement établie (intention) de satisfaction de l'intérêt général avec intelligence, appliquée à la gestion de notre maison commune (économie), à sa connaissance systémique (science) ou à sa dirrection communautaire (politique). On pourra alors s'inspirer de la manière dont une communauté chargée d'éclairer avec intelligence la stratégie militaire pour la défense de son pays, réunit des experts aux compétences différentes mais complémentaires, pour observer les objets (cf. Table 4) relevant de leur compétence propre sous tous les angles de la Sécurité (terrain ou lieu, environnement ou milieu, organisation ou appareil, activité, acteurs puis moyens), en tâter les différentes formes ou modes (générique, contextuel, fonctionnel, agile, singuliers ou critique), puis en apprécier les contenus (synoptique, relationnel, organique, opérationnel, humains et névralgique). Nos experts chargés d'éclairer l'économie de marché, la science universelle ou la politique publique, vont pouvoir ainsi observer leurs objets (cf. Tables 5, 6 et 7) sous tous les angles de la Compétitivité, du Progrès ou du Bien commun, en tâter les différentes formes, puis en apprécier les contenus.

Les Tables 4, 5, 6 et 7 ci-après montrent des exemples d'indexation de dossiers traitant chacun :

- (Table 4), de l'appareil militaire d'une entité stratégique considérée comme une «menace » (État, groupe terroriste, coalition ou tout autre sujet stratégique susceptible de faire l'objet d'un plan de dossiers du renseignement de défense), sous sa forme contextuelle (terre) et dans son contenu opérationnel (Indice 213 : situation des forces terrestres relevant de l'appareil militaire de l'entité) ;

- (Table 5), des moyens numériques d'une entité économique ou « produiit » (bien, service ou tout autre sujet économique susceptible de faire l'objet d'un plan de dossiers d'intelligence économique (Indice 521 : coopérations en matière d'outils numériques de maîtrise de l'information de l'entité) ;

- (Table 6), de l'environnement épistémologique d'une entité scientifiqque ou « chose » (fait, évènement, concept ou tout autre sujet scientifique susceptible de faire l'objet d'un plan de dossiers de la recherche scientifique (Indice 154 : écoles de pensée prônant un positivisme radical dans l'environnement épistémologique de l'entité) ;

- (Table 7), de l'activité publique d'une entité politique ou « défii » (environnement, climat, santé, éducation, numérique ou tout autre sujet politique susceptible de faire l'objet d'un plan de dossiers d'intelligence collective (Indice 332: initiatives publiques associées à l'entité touchant à l'organisation de l'activité cyber).

Table 4. Exemple d'indexation d'un dossier relatif à une «menace » objet d'intérêt du renseignement de défense.

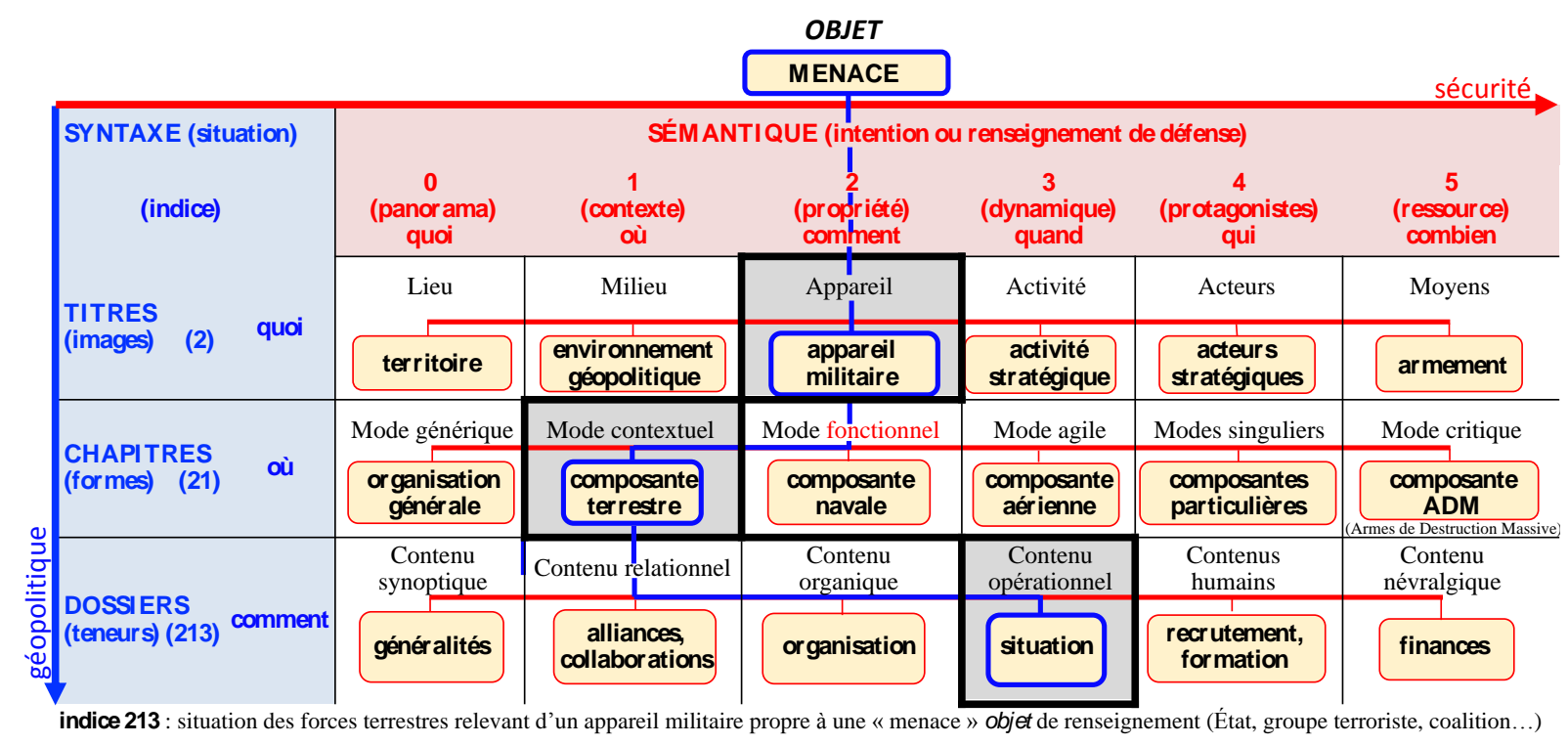


Table 5. Exemple d'indexation d'un dossier relatif à un « produit » objet d'intérêt de l'intelligence économique.

\begin{tabular}{|c|c|c|c|c|c|c|}
\hline \multirow{3}{*}{$\begin{array}{c}\text { SYNTAXE (situation) } \\
\text { (indice) }\end{array}$} & \multicolumn{3}{|r|}{$\begin{array}{c}\text { OBJET } \\
\text { PRODUIT }\end{array}$} & & & compétitivité \\
\hline & & SÉMAN & TIQUE (intention & intelligence éc & nomique) & \\
\hline & $\begin{array}{c}0 \\
\text { (panorama) } \\
\text { quoi }\end{array}$ & $\begin{array}{c}1 \\
\text { (contexte) } \\
\text { où }\end{array}$ & $\begin{array}{c}2 \\
\text { (propriété) } \\
\text { comment }\end{array}$ & $\begin{array}{c}3 \\
\text { (dynamique) } \\
\text { quand }\end{array}$ & $\begin{array}{c}4 \\
\text { (protagonistes) } \\
\text { qui } \\
\end{array}$ & $\begin{array}{c}5 \\
\text { (ressource) } \\
\text { combien }\end{array}$ \\
\hline \multirow{2}{*}{$\begin{array}{l}\text { TITRES } \\
\text { (images) }\end{array}$} & Lieu & Milieu & Appareil & Activité & Acteurs & Moyens \\
\hline & $\begin{array}{c}\text { champ } \\
\text { d'activités }\end{array}$ & $\begin{array}{c}\text { environnement } \\
\text { méthodologique }\end{array}$ & $\begin{array}{c}\text { système } \\
\text { économique } \\
\end{array}$ & \begin{tabular}{|c|} 
activité \\
economique \\
\end{tabular} & $\begin{array}{c}\text { agents } \\
\text { économiques } \\
\end{array}$ & $\begin{array}{c}\text { outil } \\
\text { numérique } \\
\end{array}$ \\
\hline \multirow{2}{*}{$\begin{array}{l}\text { CHAPITRES } \\
\text { (formes) } \\
\text { (52) }\end{array}$} & Mode générique & Mode contextuel & Mode fonctionnel & Mode agile & Modes singuliers & Mode critique \\
\hline & $\begin{array}{l}\text { outil } \\
\text { global }\end{array}$ & $\begin{array}{c}\text { outil } \\
\text { de créativité }\end{array}$ & $\begin{array}{l}\text { outil de maîtrise } \\
\text { de l'information }\end{array}$ & $\begin{array}{c}\text { outil } \\
\text { d'influence }\end{array}$ & $\begin{array}{l}\text { autres } \\
\text { outils }\end{array}$ & $\begin{array}{c}\text { outil cybernétique } \\
\text { radical }\end{array}$ \\
\hline \multirow{2}{*}{$\begin{array}{l}\text { DOSSIERS } \\
\text { (teneurs) (521) comment }\end{array}$} & $\begin{array}{c}\text { Contenu } \\
\text { synoptique }\end{array}$ & Contenu relationnel & $\begin{array}{l}\text { Confenu } \\
\text { organique }\end{array}$ & $\begin{array}{c}\text { Contenu } \\
\text { opérationnel }\end{array}$ & $\begin{array}{l}\text { Contenus } \\
\text { humains }\end{array}$ & $\begin{array}{c}\text { Contenu } \\
\text { névralgique }\end{array}$ \\
\hline & généralités & $\begin{array}{c}\text { alliances } \\
\text { collaborations }\end{array}$ & organisation & situation & $\begin{array}{l}\text { recrutement, } \\
\text { formation }\end{array}$ & finances \\
\hline
\end{tabular}

Table 6. Exemple d'indexation d'un dossier relatif à un « chose » objet d'intérêt de la recherche scientifique.

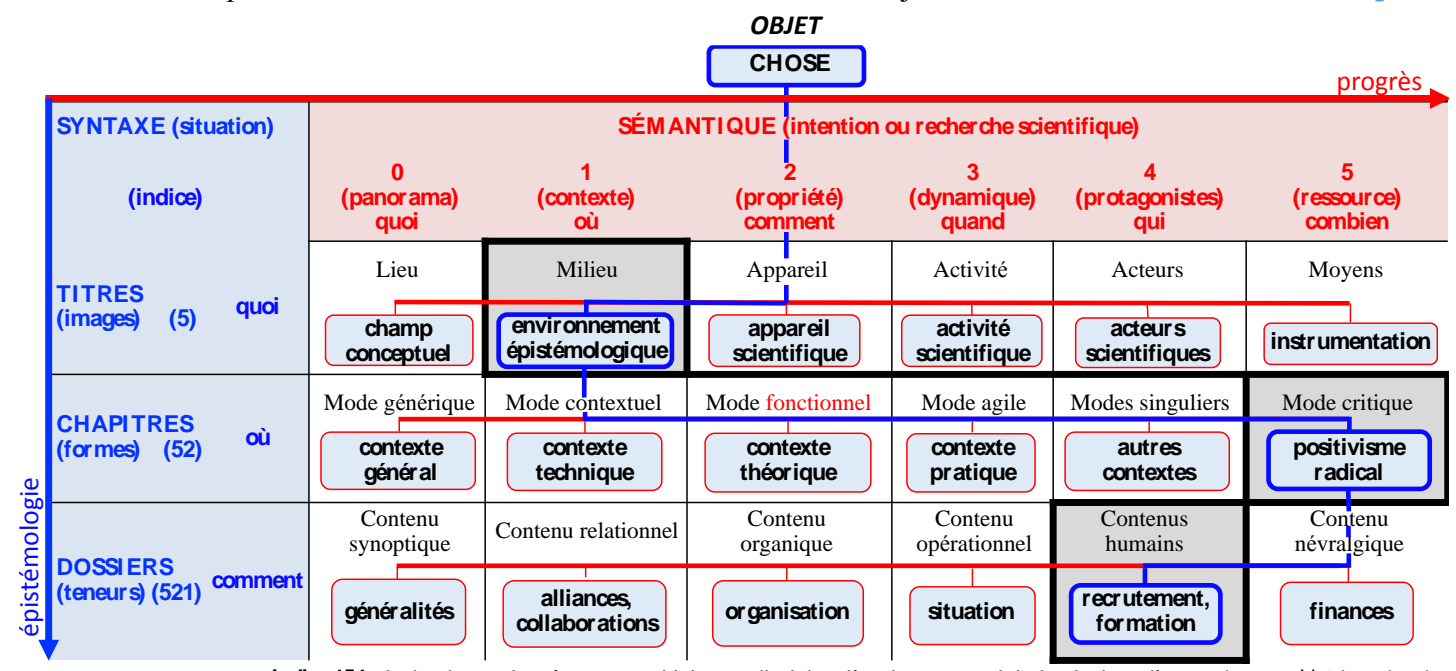

Table 7. Exemple d'indexation d'un dossier relatif à un « défi » objet d'intérêt de l'intelligence collective.

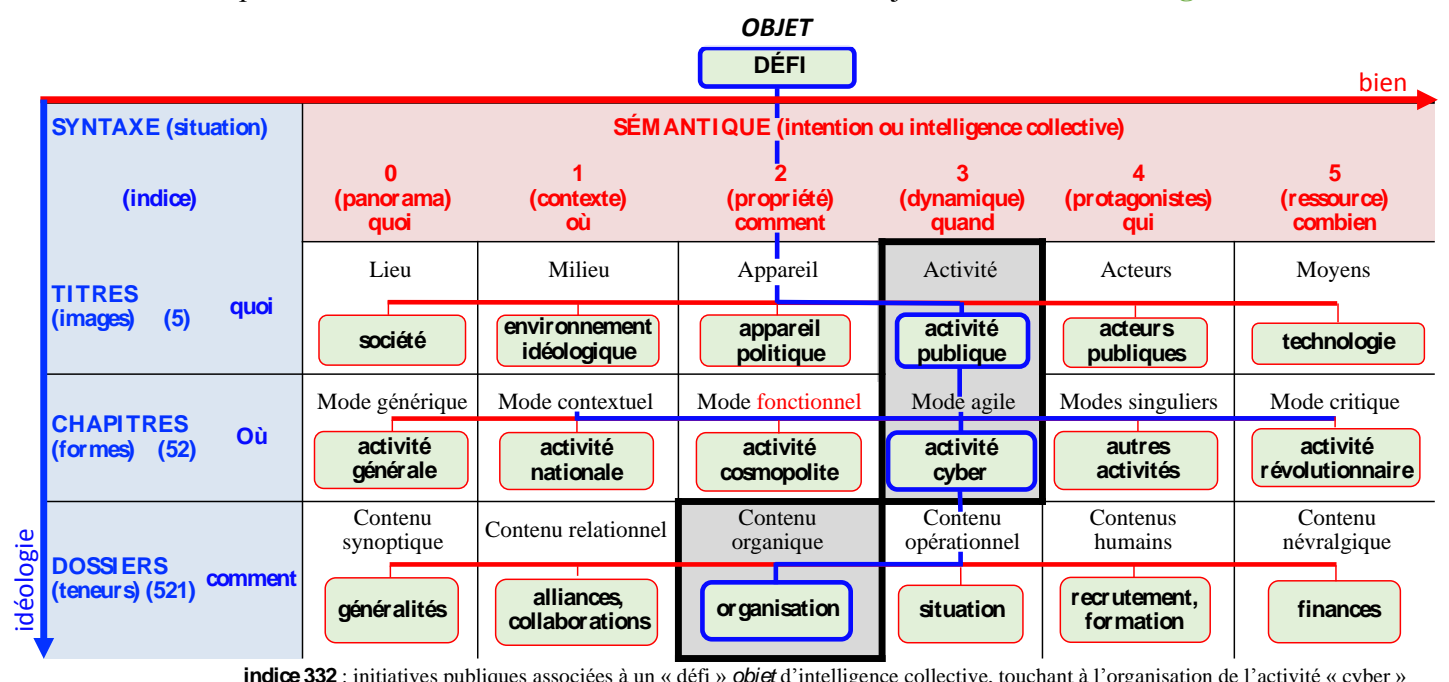




\section{ENJEUX SOCIO-ECOLOGIQUES}

Les grands défis à relever par nos sociétés modernes sont bien de nature politique au sens le plus noble du terme qui est l'art de gouverner les peuples sur la planète Terre. Pour y répondre avec discernement, il semble nécessaire d'en appeler à un changement de paradigme scientifique et politique en redonnant toute sa place à cette épistêmê aristotélicienne dont nous avons introduit plus haut quelques enseignements permettant d'aller de la stratégie à la politique en passant par l'économie et la science, dans la perspective d'un Bien commun vers lequel, selon Aristote, toutes choses doivent naturellement tendre.

Le concept souvent évoqué de «révolution technologique», dont on chercherait à anticiper les prochaines manifestations pour en exploiter les effets en préservant l'intérêt général, semble être une mauvaise façon de poser le problème du progrès scientifique. La notion de « révolution » évoque en effet une transformation profonde d'un ordre social, économique, politique ou culturel que l'on peut associer à un ou plusieurs facteurs déclenchant. Ce ne sont pas ces facteurs (ici les innovations technologiques) qui caractérisent le plus précisément la révolution dont on souhaite analyser, prédire ou anticiper les effets, mais plutôt l'ordre impacté. Il s'agit d'un ordre «culturel et cognitif », qui doit s'imposer à la préservation de l'intérêt général en balayant tout un ensemble de problèmes que l'on peut identifier à de grands défis relevant tout naturellement de l'ordre politique.

La grille conceptuelle à travers laquelle notre pensée collective va percevoir son environnement, soit les objets du monde qui l'entourent (l'univers que l'on peut considérer comme obéissant à un certain ordonnancement, le cosmos grec) en relation avec une situation en perpétuelle évolution dont nous souhaitons tirer profit dans l'intention de satisfaire notre besoin de progrès (comme celui de sécurité en matière de défense ou celui de compétitivité en matière d'économie), peut ainsi reposer sur un langage commun, dont l'usage peut être aussi simple que celui de la langue maternelle pour un enfant. Une pensée scientifique reposant sur un langage documentaire partagé par l'ensemble d'une communauté scientifique et adapté à la compréhension de systèmes dynamiques complexes tels que peuvent l'être tous les écosystèmes dans lesquels nous évoluons, peut alors être développée comme une intelligence collective.

En regard de la complexité illimitée des objets observés, cette pensée est simple. C'est là toute son incommensurable portée bénéfique sur notre capacité à comprendre les systèmes complexes. Une telle simplicité procède d'une organisation logique naturelle inspirée de nos mécanismes sensoriels dans notre système cognitif cérébral, associée à l'adoption d'une démarche itérative parcourant l'épistêmè aristotélicienne dans son intégralité, sans omettre la pratique méthodique qui donne son sens à l'action, et fait, par itérations successives, la qualité du produit avec le discernement dans l'observation et la prudence dans l'innovation. Cette simplicité, fait toute la puissance de l'intelligence collective et de la pensée analogique associée, à laquelle on peut raisonnablement penser qu'aucun artefact numérique ne pourra jamais prétendre.

L'enjeu, pour l'innovation et la maitrise de systèmes complexes, du travail de réflexion qui précède est donc de contribuer à la mise en œuvre d'un outil méthodologique d'intelligence collective. Sa traduction en actes concrets se démarque de toute sorte de discours, qu'il soit de nature économique, scientifique ou politique visant à impulser des réformes, des innovations ou des inventions. Elle est plutôt la marque d'une plaidoirie de nature stratégique, qui doit être prise comme une incitation à la maîtrise d'ouvrage d'un projet scientifique d'ensemble intégrant bien sûr la technologie, mais donnant la priorité à la dimension humaine, économique, sociale, voire éthique de l'entreprise.

\section{REFERENCES}

Aristote, trad. Tricot, J. (1959). Éthique à Nicomaque, livre I, 1094a. Éditions Les Échos du Maquis, PhiloTR, Département de philosophie Cégep de Trois-Rivières.

Arnauld, A. \& Lancelot, C. (1660). Grammaire générale et raisonnée de Port-Royal. P. Le Petit, Paris. Arnauld, A. \& Nicole, P. (1683). La Logique ou l'Art de penser. (Logique de Port-Royal) Cinquième édition revue et de nouveau augmentée, Guillaume Desprez, Paris. 
Beau, F. (2019). Le renseignement au prisme des sciences de l'information, Thèse pour obtenir le grade de Docteur de l'Université Polytechnique Hauts-de-France en Sciences de l'Information et de la Communication, présentée et soutenue le 01/04/2019 à Arenberg Créative Mine, Laboratoire DeVisu, http://www.theses.fr/2019VALE0006.

Beaufre, A. (1962). Vue d'ensemble de la stratégie, In Politique étrangère $n^{\circ} 5,27^{\mathrm{e}}$ année, p. 420.

Cavazza, F. (2019). De l'importance d'un Chief Learning Officer pour réduire la dette numérique. SYSK, https://www.sysk.fr/2019/02/11/chief-learning-officer-dette-numerique/.

Chantaraud, J.F. (2019). Le syndrome Christophe Colomb: de la difficulté d'innover. La Tribune, L'Odissée, 04/06/2019, https://www.latribune.fr/opinions/tribunes/le-syndrome-christophe-colomb-dela-difficulte-d-innover-819290.html .

Diderot, D. \& d'Alembert, J. (1751). Encyclopédie ou Dictionnaire raisonné des sciences, des arts et des métiers. André Le Breton, Paris.

Felice (de), F. (1771). Encyclopédie ou Dictionnaire universel raisonné des connaissances humaines. Yverdon.

Flichy, P. (2003). L'innovation technique: Récents développements en sciences sociales, vers une nouvelle théorie de l'innovation (Nouv. éd.). Paris: La Découverte, pp. 208, 213, 219.

Foucault, M. (1966). Les Mots et les Choses. Gallimard, Paris.

Montesquieu, C.L. "Pensées". Editeur, Desgraves L. (1950). Euvres complètes, Paris, Nagel, t. II, p. 1677.

Pelletier, Y. (2007). La dialectique aristotélicienne. Les principes clés des Topiques. Société d’Études Aristotéliciennes $2^{\mathrm{e}}$ édition, Monographies Philosophia Perennis, Bibliothèque et Archives nationales du Québec.

Schumpeter, J. (1943). Capitalisme, socialisme et démocratie. Traduction française, Paris, Payot, 1951, p. 107.

Serres, M. (2007). Les nouvelles technologies : révolution culturelle et cognitive. Conférence Quarante ans de l'INRIA, forum "Informatique et Société", Lille, www.ac-grenoble.fr/ien. bourgoinashnord/IMG/pdf_Texte_de_la_conference.pdf.

Volle, M. (2017). Le rapport entre la pensée et ses objets. www.volle.com, 9 décembre 2017. 